\title{
Hybrid Power Quality Harmonic Indices
}

\author{
Ricardo A. Lima, Claudio A. Reineri, Fernando H. Magnago \\ Facultad de Ingeniería Universidad Nacional de Río Cuarto - Argentina \\ Ruta Nacional 36 Km. 601 (5800) Río Cuarto - Córdoba - Argentina \\ e-mail : rlima@ing.unrc.edu.ar
}

\begin{abstract}
:
In this paper the most frequently used power quality indices are reviewed, and an hybrid algorithm for power quality index determination is proposed. The algorithm is based on the wavelet transform and is not limited to stationary waveforms. The wavelet transform is used to detect, classify the signals and to calculate the Total Harmonic Distortion (THD). In cases where the wavelet transform is not applicable, its detection capability allows to identify the signals and the choice of another index is done to calculate the harmonic distortion. Advantages of the new index are highlighted and its performance is compared with the traditional set of indices.
\end{abstract}

Keywords: Power quality, total harmonic distortion, wavelet analysis, fast Fourier transform, standards

\section{Introduction}

Although harmonic distortion has always existed in the past, its levels have risen recently with the increase in the use of non-linear loads. In addition, the use of sensitive electronic circuitry has grown. Moreover, the progress of utility deregulation and competition requires greater demand on the quality of power.

For this reason, in order to identify and define acceptable limits of distortion levels, standards have been established [1],[2],[3],[4] and several indices have been developed condensing the information provided by voltages and current signals from complex time and frequency domain into a number.

One of the most commonly used index is the total harmonic distortion (THD)[2], that is based on harmonic components of currents or voltages that are periodic signals. Thus, the condensation of information associated with signals that contain aperiodic, quasi periodic, or non integer multiples of power frequency terms may be misinterpreted, which happens in many industrial loads [7].

The aim of this paper is to propose a general algorithm for index determination that can be used not only for periodic signals but also for non periodic ones. The algorithm is based on wavelet transform since its capability for signal classification and identification is proven [9].

The paper is organized as follows: first, different methods used for index determination are presented, then, the proposed algorithm is described and tests results on simulated and recorded signals are presented; finally the most important conclusions are discussed.

\section{Index definition:}

Total harmonic distortion THD is the most frequently used power quality index and is defined as follows [1]:

$$
T H D=\frac{1}{V_{1}} \sqrt{\sum_{i=2}^{N} V_{i}^{2}}
$$

Where $\mathrm{i}$ represents the harmonic order, $\mathrm{Vi}$ represents the amplitude of the harmonic i, V1 denotes the amplitude of the fundamental quantity, and $\mathrm{N}$ is the maximum number of harmonics considered. These quantities are calculated through the use of the Fast Fourier Transform (FFT). Although the algorithm is simple, the main disadvantage of the THD determined by Eq. (1) is that its application is restricted to periodic signals.

To overcome the limitations of the THD, the Windowed Discrete Fourier Transform (WDFT) can be used instead. For a given signal $x$, the WDFT is defined as:

$$
W D F T=\sum_{m=0}^{L-1} x[i R+m] w[m] e^{-j \frac{2 \pi}{N} \cdot k \cdot m}
$$

where the window is defined by the window step R , and i is the ith DFT calculated; once these two parameters are selected, the frequency spectrum is defined by the sampled frequency spectrum $\mathrm{k}, \mathrm{L}$ is the window width, and $\mathrm{N}$ is the number of points used in the DFT calculation.

The WDFT allows to calculate an harmonic index in different intervals within the signals, and can be considered as periodic. Based on the WDFT the Short Term Harmonic Distortion 
(STHD) index can be defined for each window and calculated as [7]:

$$
S T H D=\frac{\sqrt{\sum_{k=2}^{N} W_{D F T} T_{[i R, k]}^{2}}}{W D F T_{[i R, 1]}}
$$

One important limitation of the STHD, is the definition of the window size [10], since the size of the window needs to be previously set up, the signals should be pre processed before in order to identify the intervals where the signals can be considered as periodic. Another suggested solution is to set the window size to one cycle which is expensive in terms of computational efforts. Moreover, the window should be set up for an integer number of cycles.

In order to improve the computational cost and to take advantages of the detection capabilities, harmonic indexes can be determined using the wavelet transform.

Wavelet transform is a linear transformation that allows time localization of different frequency components of a given signal. Consider a scaling function $\varphi(x)$ and a wavelet function $\psi(x)$, defined as:

$$
\begin{aligned}
& \varphi(x)=\sum_{k} h(k) \sqrt{2} \cdot \varphi(2 x-k) \quad \text { (4) and (5) } \\
& \psi(x)=\sqrt{2} \sum_{k}(-1)^{k} h(-k+1) \cdot \varphi(2 x-k)
\end{aligned}
$$

where coefficient $\mathrm{h}(\mathrm{k})$ is calculated as :

$$
h(k)=\langle\varphi(x), \varphi(2 x-k)\rangle
$$

Then, a family of functions can be expressed as a linear combination of the scaling and the wavelet function by dilations and translations:

$$
\begin{aligned}
& \varphi_{j, k}(x)=2^{\frac{j}{2}} \varphi\left(2^{j} \cdot x-k\right) \\
& \psi_{j, k}(x)=2^{\frac{j}{2}} \psi\left(2^{j} \cdot x-k\right)
\end{aligned}
$$

It is a linear transformation much like the Fourier transform with an important difference, however: it allows time localization of different frequency components of a given signal. Then the analyzing functions, which are called wavelets, will adjust their time-widths to their frequency in such a way that higher frequency wavelets will be very narrow and lower frequency ones will be broader.

Given a function $f(t)$, its continuous Wavelet Transform (WT) will be calculated as [9] where, $\mathrm{a}$ and $\mathrm{b}$ are the scaling (dilation) and translation (time shift)constants respectively, and psi is the wavelet function which may not be real as assumed in the above equation for simplicity.

$$
W T(f, a, b,)=\frac{1}{\sqrt{a}} \int_{-\infty}^{\infty} f(t) \psi^{*}\left(\frac{t-b}{a}\right) \cdot d t
$$

Wavelet transform of sampled waveforms can be obtained by implementing the Discrete Wavelet Transform (DWT) which is given by:

$$
\operatorname{DWT}(f, m, n)=\frac{1}{\sqrt{a_{0}^{m}}} \sum_{k} f(k) \cdot \psi^{*}\left(\frac{n-k \cdot a_{0}^{m}}{a_{0}^{m}}\right)
$$

where, the parameters a and b in Eq. (9) are replaced by $a_{0}^{m}$ and $k . a_{0}^{m}, \mathrm{k}$ and $\mathrm{m}$ being integer variables. In a standard DWT, the coefficients are sampled from the continuous WT on a dyadic grid, $a_{0}=2$ and $b_{0}=1$.

Actual implementation of the Discrete Wavelet Transform, involves successive pairs of highpass and low-pass filters at each scaling stage of the Wavelet Transform. This can be thought of as successive approximations of the same function, each approximation providing the incremental information related to a particular scale (frequency range), the first scale covering a broad frequency range at the high frequency end of the spectrum and the higher scales covering the lower end of the frequency spectrum but with progressively shorter bandwidths. Conversely, the first scale will have the highest time resolution, higher scales will cover increasingly longer time intervals.

Therefore, the Wavelet Total Harmonic Distortion $\backslash$ (WTHD) can be defined as a ratio of the sum of squares of all the weighted coefficients details of the signals to the sum of squares of the lowest frequency band approximation coefficient, and its square root is taken:

$W T H D=\sqrt{\frac{\sum_{i=1}^{m-1} \sum_{k=1}^{N_{j}} d_{i}^{2}(k)}{\sum_{k} a_{m}^{2}(k)}}$ 
where: $d_{i}$ represents the coefficient detail, $a_{m}$ the approximation coefficient, and $m$ represents the number of wavelet scales.

The index represented by Eq. (11) introduces a measure of the high frequency content of a signal as compared to its fundamental frequency and proposed in this paper, as the index in the algorithm to replace the indices of Eq. (1) and Eq. (3) in cases where these indices give an incorrect meaning or to reduce the computational effort. Therefore, an hybrid methodology is proposed in order to get the most of the index determination methods. The proposed method is explained in the next section.

\section{$3 \quad$ Proposed method}

The algorithm is based on the Wavelet Transform Coefficients WTCs, the signal is decomposed into WTCs using the Wavelet Transform. Then, decisions are made by observing the reconstruction of the high frequency signal based on the high frequency WTCs, named scale 1 coefficient.

Insignificant high frequency coefficients will imply that the signal is periodic, and the harmonic distortion is calculated using Eq. (3).

It is observed that if signals have non stationary harmonics, WTCs for scale 1 shows peaks at the start and at the end of the harmonic change. Therefore, the signal is partitioned into different segments limited by the time identified by these spikes and the calculation is made again using Eq. (11) for the different signal segments.

Similarly, it is observed that a significant peak in scale 1 WTCs is shown when transients are present. Thus, the cycle that contain the transient is not considered for that calculation.

Finally, if the scale 1 WTCs shows a significant value all over the sampled signal, that is due to the presence of interharmonics, WTHD will give an incorrect interpretation, hence, the harmonic distortion is calculated using Eq.(2)

Figure 1 shows the flowchart for the proposed algorithm based on the wavelet transform signals.

Next section contains results of simulations used to test this proposed algorithm for various signal types.

\section{$4 \quad$ Test results}

Different type of signals are considered for the analysis, such as: stationary signals, signals with non stationary harmonic components, stationary signals with short time transient phenomena, and interharmonics signals. While in principle any admissible wavelet can be used in the wavelet analysis, we have chosen to use the Daubechies 4 wavelet as the mother wavelet, with nine scale levels [11].

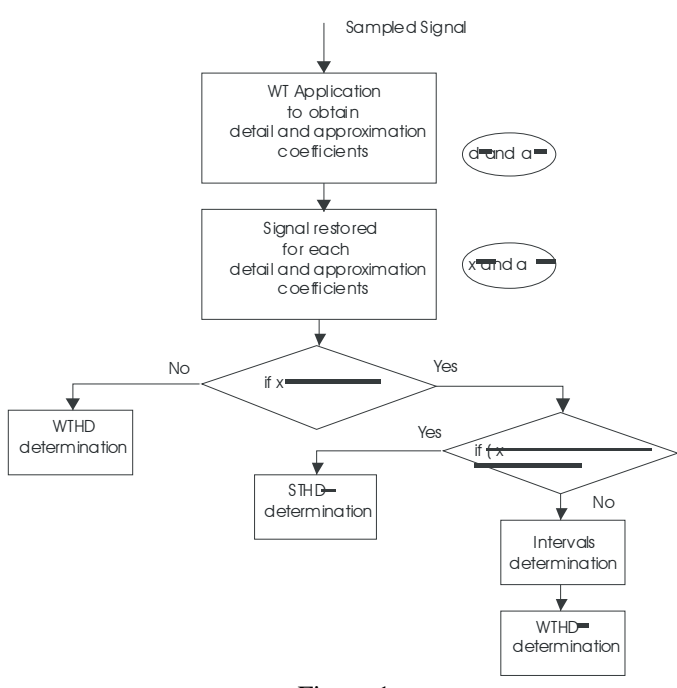

Figure 1

\section{$5 \quad$ Conclusions}

An hybrid algorithm for Total Harmonic Distortion Index calculation is proposed in this paper. The detection process is carried out using wavelet transform since its capability for signal detection and classification is proved, therefore, it is more efficient to use it instead of postprocessing the information using Fourier Transform. In some cases it will not give a correct interpretation, therefore, the algorithm uses the Fourier Transform only when Wavelet Transform is not capable to calculate the harmonic distortion, however these cases are identified through inspection of the signals related to high frequency wavelet coefficients. In addition to computer efficiency, index determination based on wavelet transform does not need an integer number of cycles to analyze the signal.

\section{Bibliography}

[1] IEEE Standard 519-1992, "Recommended Practices and Requeriments for Harmonic Control in Electrical Power Systems” - 1992.

[2] W. Reid, "Power Quality Issues - Standars and Guidelines”, IEEE Transactions on Industry Applications, Vol 32, N³, May/June 1996. 
[3] IEC 61000-3-2, "Electromagnetic compatibility EMC part 3-2: Limits - Limits for harmonic current emissions (equipment input current

less than16 A per phase)”, Edition 2.1, 2001.

[4] IEC 61000-3-4, "Electromagnetic compatibility EMC part 3-4: Limits - Limitation of emission of harmonic currents in low-voltage power supply systems for equipment with rated current greater than 16 A",

1st Edition, 1998.

[5] Sabin, D.D.; Brooks, D.L.; Sundaram, A., "Indices for assessing harmonic distortion from power quality measurements: definitions and benchmark data”, IEEE Transactions on Power Delivery, Vol. 14, No 2, April 1999, pp 489 496.

[6] IEC 1000-4-7, "Testing and Measurement Techniques - Section 7: General Guide on Harmonics and Interharmonics Measurements and Instrumentation, for Power Supply Systems and Equipment Connected thereto", 1st Edition , 1991.

[7] Jaramillo S., Heydt G., O'Neil-Carrillo E, "Power Quality Indices for Aperiodic Voltages and Currents", IEEE Transactions on Power Delivery\}, Vol 15, No 2, April 2000, pp 784790.

[8] Moo, C., Chang, Y., Mok P., "A Digital Measurement Scheme for Time-Varying Transient Harmonics", IEEE Transactions on Power Delivery, Vol 10, No 2, April 1995, pp 588-594.

[9] S. Santoso, E. Powers, W. Grady, and P. Hoffmann,"Power Quality Assessment via Wavelet Transform Analysis", IEEE Transactions on Power Delivery, Vol.11, No.2, pp. 924-930, April 1996.

[10] Gu Y., Bollen M., "Time Frequency and Time Scale Domain Analysis of Voltage Disturbances", IEEE Transactions on Power Delivery\}, Vol 15, No 4, October 2000, pp 1279-1284.

[11] I. Daubechies, Ten Lectures on Wavelets, SIAM, Philadelphia, Pennsylvania,1992. 